\title{
Exploring the Usability of a Connected Autonomous Vehicle Human Machine Interface Designed for Older Adults
}

\author{
Phillip L. Morgan ${ }^{1}$, Alexandra Voinescu ${ }^{2}$, Chris Alford ${ }^{2}$, and Praminda Caleb-Solly ${ }^{2}$ \\ ${ }^{1}$ School of Psychology, Cardiff University, 70 Park Place, Cardiff, CF10 3AT, United \\ Kingdom (morganphil@cardiff.ac.uk) \\ ${ }^{2}$ University of the West of England - Bristol, Frenchay Campus, Coldharbour Lane, Bristol, \\ BS16 1QY, United Kingdom \{alexandra.voinescu; praminda.caleb-solly; \\ chis.alford\}@uwe.ac.uk
}

\begin{abstract}
Users of Level 4-5 connected autonomous vehicles (CAVs) should not need to intervene with the dynamic driving task or monitor the driving environment, as the system will handle all driving functions. CAV humanmachine interface (HMI) dashboards for such CAVs should therefore offer features to support user situation awareness (SA) and provide additional functionality that would not be practical within non-autonomous vehicles. Though, the exact features and functions, as well as their usability, might differ depending on factors such as user needs and context of use. The current paper presents findings from a simulator trial conducted to test the usability of a prototype CAV HMI designed for older adults and/or individuals with sensory and/or physical impairments: populations that will benefit enormously from the mobility afforded by CAVs. The HMI was developed to suit needs and requirements of this demographic based upon an extensive review of HMI and HCI principles focused on accessibility, usability and functionality [1, 2], as well as studies with target users. Thirty-one 50-88 year-olds ( $M$ 67.52, three 50-59) participated in the study. They experienced four seven-minute simulated journeys, involving inner and outer urban settings with mixed speed-limits and were encouraged to explore the HMI during journeys and interact with features, including a real-time map display, vehicle status, emergency stop, and arrival time. Measures were taken pre-, during- and post- journeys. Key was the System Usability Scale [3] and measures of SA, task load, and trust in computers and automation. As predicted, SA decreased with journey experience and although cognitive load did not, there were consistent negative correlations. System usability was also related to trust in technology but not trust in automation or attitudes towards computers. Overall, the findings are important for those designing, developing and testing CAV HMIs for older adults and individuals with sensory and/or physical impairments.
\end{abstract}

Keywords: Connected Autonomous Vehicle $\cdot$ Human Machine Interface $\cdot$ Older Adults $\cdot$ Usability $\cdot$ Situation Awareness $\cdot$ Workload 


\section{Introduction}

We are moving at a rapid pace towards fully autonomous vehicles that handle all driving related functions without the need for user intervention. Level 5 fully connected autonomous vehicles (CAVs) are defined by SAE International [4] as systems that can handle 'the full-time performance by an automated driving system of all aspects of the dynamic driving task under all roadway and environmental conditions that can be managed by a human driver', whereas Level 4 highly automated vehicles are similar but only have such system capabilities for some driving modes and may require human intervention for some non-driving related actions. Both levels assume that the user is not an active driver and can therefore have eyes and mind off the road. This means they will not require driving input devices such as a steering wheel and pedals. Instead, user interaction with Level 4 and 5 CAVs will be through human-machine interface (HMI) dashboards providing vehicle-related (e.g., speed, time/distance to destination, local area information) and other features and functions (e.g., infotainment). Level 4 CAV HMIs may also offer some interactive features such as the ability to personalize journeys (e.g., an unplanned stop or deviation) en route. [4-6] It is therefore vital for human factors specialists, designers, engineers, and programmers - to develop effective CAV HMIs tailored to the needs and requirements of those using them and for a high degree of system usability. This will likely enhance factors such as trust, positive attitudes towards automated systems, and ultimately adoption and continued use of CAVs. The current paper presents findings relating to the evaluation of a prototype CAV HMI designed for older adults and/or individuals with sensory and/or physical impairments. Both populations are likely to be early adopters of Level 4 and 5 CAVs due the significant and increased mobility options that they will offer. The key focus is on HMI usability and relationships with important factors such as situation awareness (SA), task load, attitudes towards computers, and, trust in technology and automation.

A recent review by [2] (see also [1]) synthetized best practice design principles to inform the development of CAV in-vehicle HMIs for use by older adults and individuals with sensory and/or physical impairments that would prevent them from driving or mean that driving ability is impaired. They organized these into five themed areas including general principles and ageing-related factors that could affect HMI accessibility and usability, and inform functionality and adaptability requirements. We discuss these below and give examples that relate to the design of CAV in-vehicle HMIs for use by our target populations with a key focus on usability principles.

General principles put forward by [7] emphasize the importance of factors such as: providing informative feedback (e.g., verification - 'confirm', 'cancel' - of a chosen action such as destination); consistency (e.g., location and grouping of related features such as speed and time to destination); allow easy error handling (option to reverse actions such as when accidentally activating a system stop button); and reduce memory demands (e.g., easy access to important information such as current location, destination, and any stops). [8] extended these to include e.g.: striving for a good match between the system and the real world (e.g., meaningful icons displayed in a logical manner - such as a battery for energy status); ensuring easy access to system status information (e.g., vehicle status in terms of tyres, brakes, engine and network), simplicity (e.g., only display information that is absolutely necessary, avoid display clutter), and to always aim to establish standards and conventions that can be adhered 
to. [9] further extended these with a key focus on human cognitive capabilities and limitations relating to: perception (e.g., ensure display features are legible and/or audible), attention (e.g., avoid overloading one or more senses and/or resources, see [10]), memory (similar to recommendations by [7]); mental models (similar to Nielsen's match between the system and real world principle); and SA (e.g., include information about current and future status - e.g., dynamic real time map).

Despite these informative and well utilized general recommendations, very few take into account needs and requirements of specific population sectors, such as older adults for specific contexts of use, such as CAVs. These are important considerations and are likely to affect HMI accessibility, functionality and adaptability. For example, accessibility requirements for such individuals include (e.g., [11]): using touchscreen devices with voice interaction and ensuring displayed information is as simple to understand and uncluttered as possible. Key functionality examples include visual and auditory collision warnings, night vision enhancement and health and stress monitoring (e.g., [12], [13]). HMI adaptability refers to the ability to personalize features (e.g., text and image size/types) settings (e.g., option to display more or less information and have more or less steps to complete an action, and modes of interaction (see [1]).

Over and above the factors outlined above, $[1,2]$ stressed the importance of the usability of CAV in-vehicle HMIs amongst older adults and/or individuals with sensory and/or physical impairments as a key determinant (as well as e.g., trust in CAVs) of their success, continued and future use (see also [14], [15], [11]). Morgan et al. ([1], p. 328) defined CAV HMI usability as '...aspects of the HMI including: learnability; efficiency; memorability: error handling; and satisfaction (linked with likelihood of continued use)'. Many of the recommendations are based on bodies of work focused on design principles for older adults ([16], [17]) and can be divided into those that are important in terms of physical, sensory and cognitive factors. Physical principles include: avoiding pointing and dragging features that require a certain degree of agility, incorporating voice interaction where possible, and avoiding long action sequences ([17], [18], [19], [20]). In terms of sensory impairments that are much more likely amongst older adults ([21]), visual recommendations include: avoidance of dynamic text presentation (e.g., scrolling updates), spacing buttons to be at least 3.17-mm apart; and using large enough buttons (15-20-mm) ([17], [22], [23], [24]). There are also many auditory recommendations, although auditory functionality was not feasible within the prototype HMI tested and reported on in the current paper but these will be revisited in the limitations section and we are implementing them for the next iteration of the HMI.

When designing usable HMIs for older adults, we also need to take into account possible cognitive limitations relating to perception, attention and inhibition, and, short and long term memory [25]. Generally, displays should be designed to: be as simple to understand and learn as possible (i.e., reduce memory load); be relatively free of attention grabbing distracting stimuli (apart from those that are essential; should have easily distinguishable information; and should be void of screen clutter $[16,17]$. Focusing on the ability to remember things over the short-term which is limited (e.g., [26]) in terms of duration and capacity, recommendations include: avoiding long complex instructions; having clearly labelled features; using design features that map onto conceptual or mental models (e.g., 'green' for system okay), and using pictorial aids where possible (e.g., visual representation of fuel status as well as a numeric value). We also know that long-term memory can decline as we age (e.g., [11], [27], [28]) and 
HMI design recommendations include: offering practice to learn task procedures; incorporating simple intuitive steps to perform tasks; and implementing features to support fallible prospective memory (e.g., reminder cues to perform particular actions). Overall, there are many HMI design principles concerning usability that can inform the design of in-vehicle HMIs for use amongst older adults and/or younger individuals with physical, sensory and cognitive impairments. Yet, and to our knowledge, no studies have implemented and tested these within CAVs and thus the current paper is novel.

We acknowledge as with any prototype HMI, there will be issues and limitations especially because we are also using a very novel context (i.e., fully autonomous driving). Due to these and other possible drawbacks, it is important that we not only test usability, but also look at other factors that are likely to be influenced by and related to it such as SA (e.g., [29], [30], [31]) and task load ([32], [33], [34] [35]). System SA involves effectively perceiving the current situation (e.g., status, dynamics, environment attributes), comprehending information provided (e.g., using pattern recognition, evaluation) and, most difficultly, being able to project future status. Second, cognitive/mental load is related to mental processing resources including attention and can be inflated by e.g., tasks (or task features) that are complex, busy environments, and stressful situations (e.g., [36]). Both SA and workload are sensitive to system usability ([37] [38], [39]) and all are related to vehicle automation [38].

Finally, HMI usability is likely to be related to attitudes concerning computers [40] [16] and trust in technology and automation ([41], [42], [43]) and these should be considered. Concerning older adults, they are more likely to use and develop trust with HMIs that are designed according to their abilities and needs ([18], [44], [45]).

\section{Current Study}

The study was conducted to examine the usability of a prototype CAV HMI designed for older adults and/or individuals with sensory and/or physical impairments based upon a range of the best practice principles discussed above. We tested HMI usability ([3]) as well as SA ([46]) and cognitive load ([47]) during a simulator study where participants experienced fully automated driving scenarios with the HMI. They were encouraged to explore HMI features and functions during journeys. We also measured attitudes towards computers and trust in technology and computers an automation given that these might also be related to HMI usability. There were a number of hypotheses. First, higher system usability would be positively correlated with SA as a more usable system will better support perception, comprehension, and projection (e.g., [29]). Second, higher system usability will be positively correlated with task load as a more usable system will place fewer demands on cognitive load (e.g., [35]). Third, if the HMI is deemed highly usable, SA will improve with increased experience whilst cognitive load will decrease. Finally, there will be positive correlations between system usability and attitudes towards computers and trust in technology and automation.

\section{$2 \quad$ Method}

Participants. Thirty-one adults, aged 47-88 $(M=67.52, S D=7.29$, three younger than $60)$, volunteered. This provided an adequate sample to detect medium-large effect sizes (Cohen's $f=.25-.4$ ) with power of .8 [48] on main measures. Most were male $(61.3 \%)$. 
Inclusion criteria were: fluency in English language and comprehension and those with mild to moderate visual or hearing impairments could take part. Thirty had corrected vision and four had corrected hearing. One participant had a significant visual impairment. Despite this, all participants were highly functional. Those with significant health conditions (e.g. epilepsy, neurological impairments, coronary issues) were not permitted to take part. Participants received a $£ 20$ voucher to cover travel costs.

Design. A cross-sectional correlational design was adopted to investigate relationships between outcomes, including system (HMI) usability, SA, cognitive workload, and trust in automation and computers. Pearson's $r$ correlations were employed as a measure of effect size, with $\leq .10$ indicating a small effect size, .30 a medium effect, and $\geq .50$ a large effect [48]. For comparisons between journey order (counterbalanced) and in case of differences due to task load and SA, a repeated measures design was employed. Journey order was counterbalanced with a Latin square.

Materials. The prototype HMI (Fig 1) design was informed by an extensive literature review [2] that synthesized best recommendations for older people (e.g. reduce clutter, use large icons, use icons that are highly intuitive). The HMI was delivered on a 12.9 inch iPad Pro with ED backlit display and $P$ PS technology; retina display; 2732 x 2048 resolution at 264 pixels per inch, and fingerprint resistant coating. The main screen had the following features and functionality (Fig 1): Google-based navigation map with active zoom and vehicle position tracking; vehicle status (drill-down to e.g., battery status, tyre pressure); fuel (drill-down to energy status); arrival time (with button press to change information layout in real time); speed (mph: not adaptable); date and time; and stop (two options: one to confirm and the other to cancel and return to main screen).

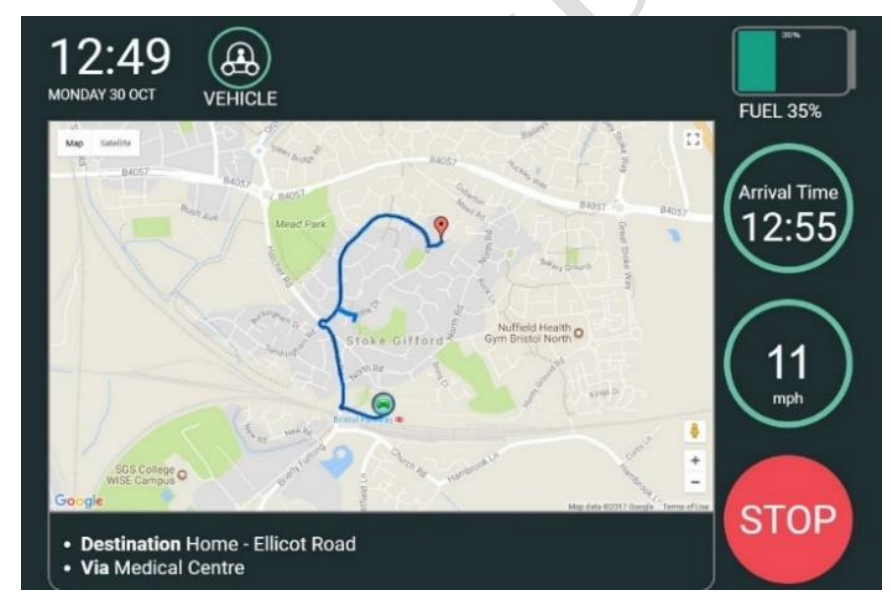

Fig 1. Prototype HMI main screen

The simulator was an adapted Lutz pod (Fig 2) designed and supplied by Transport System Catapult (TSC). The steering wheel was removed. Scenarios were presented via an Alienware I7, 2.60GHz processor laptop at a refresh rate of $60 \mathrm{~Hz}$ and displayed on a Samsung 60-inch 4K 3840 x 2160 pixels Ultra HD Smart TV situated in front of the 
pod taking up the entire windscreen area. The scenarios were recorded using 3 x GoPro Hero 4 cameras. The camera rig was mounted on a static support designed by Bristol Robotics Laboratory. The recordings were filmed while driving a Hybrid vehicle in electric mode that largely mimics the view that would be perceived from the Lutz pod.

Twelve journeys were filmed, mainly on public roads in and around Bristol (UK) city during the summer. Each route was planned using Google Maps and a route planner, and included a 2-minute pre-planned stop. Two journey types were recorded: short (7minutes including stop) and long (12-minutes including stop). Four were selected (two short, two long) which gave a good representation of driving within inner and outer urban Bristol settings, with a mixture of road types, speed limits ranging from 20-40$\mathrm{mph}$, a blend of road infrastructure (e.g., traffic lights, crossings, roundabouts), and varied scenes (highly built-up to outer city suburbs with green spaces). An example journey was travelling from a railway station to home via a medical center.

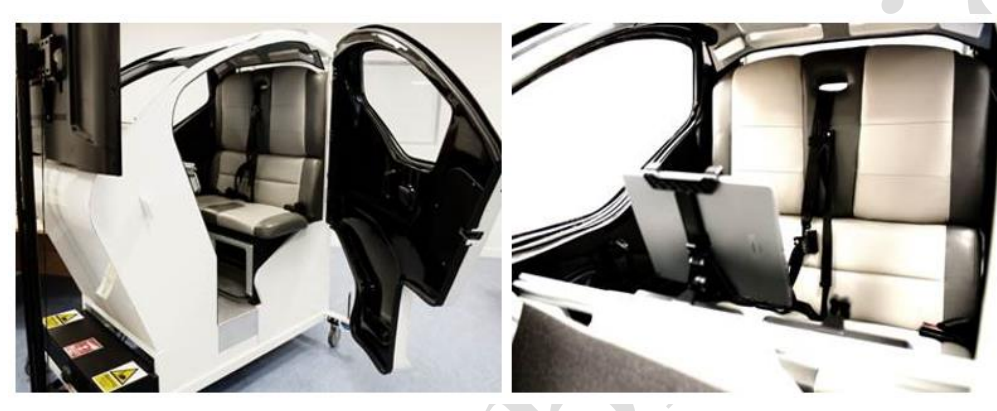

Fig 2. Exterior (left) and interior (right) of Lutz Pod simulator

Key measures linked to the aims of the current paper included the:

- System Usability Scale / SUS [3] for usability and user experience on factors such as need for support, training, and complexity. Higher scores reflect better system usability. - Raw NASA Task Load Index / TLX [47] for subjective post-journey workload on dimensions including mental, physical and temporal demand, and performance, effort and frustration level. Higher scores indicate an increased estimate of overall workload. - Situation Awareness Rating Technique / SART [46] for post journey SA on dimensions such as situation instability, complexity and variation, concentration and division of attention, and arousal. Lower scores represent increased SA.

- Attitudes Toward Computers Questionnaire / ATCQ [49] to assess comfort, efficacy, gender equality, control, interest dehumanization and utility (the belief that computers are useful). Each dimension has 5 or 6 items and answered using 5-point Likert scales; lowers scores reflect more negative attitudes towards computers.

- Checklist for Trust between People and Automation / CTPA [50]. Contains 12 questions on aspects such as dependability and reliability of the system and suspicion and confidence. Higher scores imply increased system dependability and ratability.

- General Trust Scale / GTS [51]. Contains seven questions with 7-point Likert scales and a higher overall score represents higher trust in technology.

- A demographic questionnaire was also included with questions on e.g., age, gender, qualifications, and driving status (e.g., current driver, past driver, never driven). Other measures were used but discussion is beyond the scope of the current paper. 
Procedure. Participants attended an induction 'In-Vehicle Participant Workshop' held at least two weeks before the experiment. The purpose was to inform them about the project aims, timescale and to gather information relating to e.g., expectancies about CAVs (e.g., journey types, likelihood of using, HMI design features). It also served to try and keep expectancies about CAVs to a similar level. The experiment had 4 phases (see below) and lasted 165-240-minutes, depending on needs, breaks and questions.

Pre-test. Participants were provided with a general briefing relating to the study. Then, they completed the following scales and measures: self-screening questionnaire, demographic questionnaire, and pre-test versions of the SSQ, PANAS and NASA TLX.

Test. Participants were sat in the simulator and experienced the four virtual journeys on the screen in front while interacting with the HMI. The first journey was practice for familiarization and initial usability testing. At the beginning of each journey, participants read the instruction sheets that contained an overview of journey tasks. During the first journey, the experimenter sat next to the participant, and set up the HMI (e.g., destination, planned stop). For the next three journeys, participants were seated alone and received journey specific instructions at the start of each journey on instruction sheets with information about the scenario, destination, set-up instructions and tasks to perform in order to have a controlled assessment of interaction with the HMI. After each journey, they completed NASA TLX, PANAS and SART scales.

Post-test. Participants completed the SSQ, CTBA, SUS, and PQ scales, followed by computerized cognitive ability. They were then debriefed.

Follow-up. GTS, ATCQ, CFQ and ZKPQ were completed at home within 48-hours.

\section{$3 \quad$ Results and Discussion}

HMI Usability and SA

Table 1 displays Pearson $r$ correlation coefficients for HMI usability and SA across each of the four journeys (including journey 1 - practice). None of the correlations were significant. Thus, our first hypothesis was not supported in that it seems that there are not any associations between SA and the perceived usability of our prototype HMI. It is however noted that the prototype HMI is quite basic at this stage and there are not many demands on participants to e.g., comprehend complex information or project and predict future sates. Future iterations of the HMI are likely to have increased functionality and adaptability that might be better supported by increased SA.

Table 1. Correlation coefficients (Pearson $r$ ) for usability and SA.

\begin{tabular}{|l|c|c|c|c|}
\hline & Journey 1 & Journey 2 & Journey 3 & Journey 4 \\
\hline & \multicolumn{4}{|c|}{ Situation Awareness } \\
\hline System & $M 3.03$ & $M 4.65$ & $M 4.34$ & $M 1.04$ \\
Usability $\quad(M$ & $-.16(\mathrm{~ns})$ & $.02(\mathrm{~ns})$ & $-.02(\mathrm{~ns})$ & $.11(\mathrm{~ns})$ \\
$77.90)$ & & & \\
\hline
\end{tabular}

Note. Ns $=$ non-significant. 
We also assessed whether SA differed between journeys. A repeated measures analysis of variance (ANOVA) using Pillai's trace revealed a significant main effect, $V$ $=0.41, F(3,18)=4.29, p<.05$, supporting our third hypothesis. Post hoc comparisons using Bonferroni corrections identified significantly higher SA following journey 1 versus journey 4 . There were no other significant differences. Perhaps longer scenarios are required to unearth consistent differences between all journeys.

\section{HMI Usability and Task Load}

Table 2 displays correlation coefficients for HMI usability and task load across journeys. Subjective workload is negatively correlated with HMI usability such that the lower the judged usability the higher the reported cognitive task load. This supports our second hypothesis although challenges our interpretation of the non-significant usability and SA relationships as it suggests the HMI was complex enough to invoke increased workload ratings. However, this finding may have emerged due to e.g., the relative lack of familiarity with the HMI and its features and functions following only one practice journey as well as occasional responsiveness issues with some of the HMI functions that were likely due to technical networking and processing issues.

Table 2. Correlation coefficients (Pearson $r$ ) for usability and task load.

\begin{tabular}{|l|c|c|c|c|}
\hline & Journey 1 & Journey 2 & Journey 3 & Journey 4 \\
\hline & \multicolumn{4}{|c|}{ Task Load } \\
\hline System & $M 5.20$ & $M 5.78$ & $M 6.31$ & $M 6.48$ \\
Usability & $-.46^{* *}$ & $-.40^{*}$ & $-.51^{* *}$ & $-.36^{*}$ \\
\hline Note. ${ }^{*} \mathrm{p}<.05,{ }^{* *}, \mathrm{p}<.01$.
\end{tabular}

Next, we assess whether task load differed between journeys. A repeated measures ANOVA using Pillai's trace revealed a non-significant main effect, $V=0.21, F(4,25)$ $=1.70, p>.05$, suggesting that the usability of the HMI did not affect task load between journeys. We predicted that workload would decrease across journeys but it may have been that it was low enough within the practice journey and did not get much lower.

\section{HMI Usability and Trust and Attitudes Towards Computers and Automation}

Table 3 displays Pearson $r$ correlation coefficients for usability and: attitudes towards computers, general trust in technology, and trust in automation. Attitudes towards computers and trust in automation were not related with usability, although there was is significant positive correlation with general trust in technology such that, and as predicted, those with higher trust also had the highest usability ratings. It was less surprising to find a non-significant relationship between usability and trust in automation, as they were rating HMI usability and not the automated journeys.

Table 3. Correlation coefficients (Pearson $r$ ) for usability, attitudes towards computers, general trust in technology, and trust in automation.

\begin{tabular}{|l|c|c|c|}
\hline & $\begin{array}{c}\text { Attitudes Towards } \\
\text { Computers }(M \\
133.31, S D 13.42)\end{array}$ & $\begin{array}{c}\text { General Trust in } \\
\text { Technology }(M \\
22.59, S D 3.82)\end{array}$ & $\begin{array}{c}\text { Trust in } \\
\text { Automation }(M \\
60.03, S D 12.58)\end{array}$ \\
\hline System Usability & $.12(\mathrm{~ns})$ & $.40^{*}$ & $.05(\mathrm{~ns})$ \\
\hline
\end{tabular}

Note. Ns $=$ non-significant, $* \mathrm{p}<.05$. 


\section{$4 \quad$ Limitations}

There are limitations within the study that require future attention. First, the prototype HMI involved a touchscreen interface with visual features and functions and no audio (e.g., 'ask CAV', 'CAV speaks') capabilities. Many have noted that HMIs with auditory capabilities not only tend to be considered more usable (e.g., [17], [11]) but also that they can be more adaptable. These are highly desirable factors when designing for populations with a higher propensity of visual sensory impairments. When incorporating auditory capabilities, a number of recommendations need to be considered such as using sound signals that are a minimum of $60 \mathrm{~dB}$ but not too loud, and, ensuring that sounds can be discriminated using a $500-1000 \mathrm{~Hz}$ frequency range (e.g., [11], [51], [52]). Second, the prototype interface offered little in the way of adaptability which we know can enhance usability, especially amongst older adult users [53], especially in relation to features such as: modifying textual information (e.g., ability to change font, increase/decrease font size, etc.); having zoom functions (noting the map feature did); and being able to switch between visual and auditory information updates. Third, whilst we tested a respectable sample size of adults with quite a variation in age (e.g., 28 of the 31 were aged between 60-83), gender, and background, many were self-selecting despite our recruitment protocols (i.e., advertisements sent via our project collaborators AgeUK and other associations. Thus, many may have chosen to take part because of a desire to find out more about CAV technology. Finally, we acknowledge the importance of other individual difference factors such as cognitive ability that are likely to have added to the variance in data. We did collect such data, although it is beyond the scope of the current paper to report on it (though see [54]).

\section{$5 \quad$ Implications}

There are a many implications that warrant future consideration. First, we have successfully demonstrated that our mainly older adult participants can interact with an in-vehicle HMI used in a Level 5 CAV simulator to a degree that they can assess its usability. Second, our study offers insight on the way in which some individual variables relate to usability. For example, general trust in technology, as a stable trait, is associated with a positive HMI usability scores, while other individual variables, such as trust in automation and attitudes towards computers are not associated likewise. Third, task-related measures like subjective workload negatively correlate with HMI usability. This suggests that the more a situation is perceived as demanding in terms of interaction between the human and the system (i.e., HMI), the less likely it is that usability will be rated positive. Such a finding might indicate a preference of older adults for simple, easy, less complex HMIs, although it is noted that some issues with screen responsiveness may have led to participants perceiving the HMI to be more cognitively taxing than intended. Fourth, the level of SA in simulated journeys did not correlate with usability scores, although, our results point out that at the beginning of the trial, for journey 1, participants were more likely to display increased SA and actively screen the environment compared to the last journey when their SA had decreased significantly. Finally, taken together our results give a unique insight into how a sample of older adults' cognitive and perceptual characteristics relate to their 
experience of CAV in-vehicle HMI usability, and how HMI features, as well as journey-related features are equally important. Overall, our older participants enjoyed the simulated journeys and considered the experience as being novel and real. Though, at the beginning of journeys they had a tendency to focus attention more on the road, as actually being present and judging the ability of the "autonomous" driver to drive safely, and less on the HMI. Future studies might consider investigating user behavior on longer journeys that will give participants longer time to interact with the HMI, and see whether driving related measures (e.g. safe and trust) will impact HMI interaction.

Acknowledgments. The research is part of an Innovate UK research project FLOURISH: Empowerment through Trusted Secure Mobility (2016-2019: http://flourish mobility.com/). We thank our collaborators at Designability (Jessica Ridgers, Keir Haines, Hazel Boyd) for designing the HMI and at TSC (Alan Nettleton, Alan Peters, and Roland Guichard) for programming the prototype HMI.

\section{References}

1. Morgan, P.L., Voinescu, A., Williams, C., Caleb-Solly, P., Alford, C., Shergold, I., Pipe, A.: An Emerging Framework to Inform Effective Design of Human-Machine Interfaces for Older Adults Using Connected Autonomous Vehicles. In: International Conference on Applied Human Factors and Ergonomics, 325--334. Springer, Cham, (2017)

2. Morgan, P., Caleb-Solly, P., Voinescu, A., Williams, C.: Literature Review: HumanMachine Interface. Project report., UWE Bristol (2016)

3. Brooke, J.: System usability scale (SUS): a quick-and-dirty method of system evaluation user information. Digital Equipment Co Ltd, 43, Reading, UK (1986)

4. International, S.: U.S. Department of transportation's new policy on automated vehicles adopts SAE International's levels of automation for defining driving automation in on-road motor vehicles. (2016)

5. Morgan, P., Alford, C., Parkhurst, G.: Handover issues in autonomous driving: A literature review. Project Report. pp. 1-18. University of the West of England, Bristol, UK (2016)

6. Kun, A.L., Boll, S., Schmidt, A.: Shifting gears: User interfaces in the age of autonomous driving. IEEE Pervasive Computing 15, 32--38 (2016)

7. Shneiderman, B.: Designing the user interface, Strategies for effective human computer interaction. Addison Wesley Publishing Company, Boston, MA, (1987)

8. Nielsen, J., Mack, R.: Usability inspection methods. John Wiley \& Sons, New York, NY (1994)

9. Wickens, C.D., Lee, J., Liu, Y., Becker, S.G.: An introduction to human factors engineering. Pearson, New Jersey, NJ (2004)

10. Wickens, C.D.: The multiple resources model of human performance: Implications for display design. Univ At Urbana, Illinois (1984)

11. Fisk, A.D., Czaja, S.J., Rogers, W.A., Charness, N., Sharit, J.: Designing for older adults: Principles and creative human factors approaches. CRC press, Florida, FL (2009)

12. Eisses, S.: ITS Action Plan. RappTrans. . European commission D4 Final Report (2011)

13. Emmerson, C., Guo, W., Blythe, P., Namdeo, A., Edwards, S.: Fork in the road: In-vehicle navigation systems and older drivers. Transportation Research Part F: Traffic Psychology and Behaviour 21, 173-180 (2013)

14. Bruder, C., Blessing, L., Wandke, H.: Adaptive training interfaces for less-experienced, elderly users of electronic devices. Behaviour \& Information Technology 33, 4-15 (2014) 
15. Young, K.L., Koppel, S., Charlton, J.L.: Toward best practice in human machine interface design for older drivers: a review of current design guidelines. Accident Analysis \& Prevention 106, 460--467 (2017)

16. Czaja, S.J., Charness, N., Fisk, A.D., Hertzog, C., Nair, S.N., Rogers, W.A., Sharit, J.: Factors predicting the use of technology: findings from the Center for Re-search and Education on Aging and Technology Enhancement (CREATE). Psychology and Aging 21, 333--352 (2006)

17. Farage, M.A., Miller, K.W., Ajayi, F., Hutchins, D.: Design principles to accommodate older adults. Global Journal of Health Science 4, 2--25 (2012)

18. Darejeh, A., Singh, D.: A review on user interface design principles to increase software usability for users with less computer literacy. Journal of Computer Science 9, 1443--1450 (2013)

19. Dieudonné, V., Mahieu, P., Machgeels, C.: INPH, a navigation interface for motor-disabled persons. Proceedings of the 15 th Conference on l'Interaction Homme-Machine, pp. 202-$205(2003)$

20. Page, T.: Touchscreen mobile devices and older adults: a usability study. International Journal of Human Factors and Ergonomics 3, 65--85 (2014)

21. Dickinson, A., Arnott, J., Prior, S.: Methods for human-computer interaction research with older people. Behaviour \& Information Technology 26, 343--352 (2007)

22. Morrell, R.W., Echt, K.V.: Designing written instructions for older adults: Learning to use computers. In: Fisk, A.D., Rogers, W.A. (eds.) Handbook of human factors and the older adult. Academic, New York, NY (1997)

23. Rubin, G.S., Roche, K.B., Prasada-Rao, P., Fried, L.P.: Visual impairment and disability in older adults. Optometry \& Vision Science 71, 750--760 (1994)

24. Watson, D.G., Maylor, E.A.: Aging and visual marking: Selective deficits for moving stimuli. Psychology and Aging 17, 321--339 (2002)

25. Caleb-Solly, P.: Person-environment interaction. In: F., Florez-Revuelta, F. A., Andre, A. (eds.) Active and Assisted Living: Technologies and Applications., pp. 143-162. IET (2016)

26. Baddeley, A.: Working memory: theories, models, and controversies. Annual Review of Psychology 63, 1--29 (2012)

27. Glisky, E.L.: Changes in Cognitive Function in Human Aging. In: Riddle, D.R. (ed.) Brain Aging: Models, Methods, and Mechanisms. CRC Press, FL (2007)

28. Spencer, W.D., Raz, N.: Differential effects of aging on memory for content and context: A meta-analysis. Psychology and Aging 10, 527--539 (1995)

29. Endsley, M.R.: Toward a theory of situation awareness in dynamic systems. Human Factors 37, 32--64 (1995)

30. Endsley, M.R.: Situation awareness misconceptions and misunderstandings. Journal of Cognitive Engineering and Decision Making 9, 4--32 (2015)

31. Morgan, P., Patrick, J.: Designing interfaces that encourage a more effortful cognitive strategy. In: Proceedings of the 54th Annual Meeting of the Human Factors and Ergonomics Society, pp. 408--412. (2010)

32. Moray, N.: Models and measures of mental workload. In: Moray, M. (ed.) Mental Workload. Springer, Boston, MA (1979)

33. Parasuraman, R., Hancock, P.: Adaptive control of workload. In: Hancock, P.A., Desmond, P.E. (eds.) Stress, workload, and fatigue, pp. 305-320. Erlbaum, Mahwah, NJ (2001)

34. Tsang, P., Wilson, G.F.: Mental Workload. In: Salvendy, G. (ed.) Handbook of Human Factors and Ergonomics, pp. 417-449. Wiley, New York, NY (1997)

35. Wickens, C.D.: Multiple resources and mental workload. Human Factors 50, 449--455 (2008)

36. Wickens, C.D., Hollands, J.G., Banbury, S., Parasuraman, R.: Engineering Psychology \& Human Performance. Routlege, New York, NY (2015) 
37. Endsley, M.R., Jones, D.G.: Designing for situation awareness: An approach to usercentered design. CRC press, Boca Raton, FL (2016)

38. Walker, G.H., Stanton, N.A., Salmon, P.M.: Human Factors in Automotive Engineering and Technology. Ashgate, Aldershot, UK (2015)

39. Park, P.C.: Comparison of subjective mental workload assessment techniques for the evaluation of in-vehicle navigation systems usability. In: Towards the New Horizon Together. Proceedings of the 5th World Congress On Intelligent Transport Systems. (1998)

40. Agarwal, R., Karahanna, E.: Time flies when you're having fun: Cognitive absorption and beliefs about information technology usage. MIS quarterly 24, 665--694 (2000)

41. Ekman, F., Johansson, M., Sochor, J.: Creating appropriate trust for autono-mous vehicle systems: A framework for HMI Design. Transportation Research Board 95th Annual Meeting (No. 16-3268), Washington, D.C. (2016)

42. Hoff, K.A., Bashir, M.: Trust in automation: Integrating empirical evidence on factors that influence trust. Human Factors 57, 407--434 (2015)

43. Mirnig, A.G., Wintersberger, P., Sutter, C., Ziegler, J.: A framework for analyzing and calibrating trust in automated vehicles. In: Proceedings of the 8th In-ternational Conference on Automotive User Interfaces and Interactive Vehicular Applications Adjunct pp. 33--38. ACM, (2016)

44. Aula, A.: Learning to use computers at a later age. HCI and the Older Population 3, 1--4 (2004)

45. Tsai, H.Y.S., Shillair, R., Cotten, S.R., Winstead, V., Yost, E.: Getting grand-ma online: are tablets the answer for increasing digital inclusion for older adults in the US? Educational Gerontology 4, 695--709 (2015)

46. Selcon, S.J., Taylor, R.M.: Situational Awareness Rating Technique (SART): The Developement Of A Tool For Aircrew Systems Design. . In: Proceeding of the AGARD AMP Symposium on Situational Awareness in Aerospace Operations, CP478. NATO AGARD., (1990)

47. Hart, S.G., Staveland, L.E.: Development of NASA-TLX (Task Load Index): Results of empirical and theoretical research. Advances in Psychology 52, 139--183 (1988)

48. Cohen, J.: Statistical Power Analysis for the Behavioral Sciences. La Wrence Erlbaum Associates, Publishers, Hillsdale, New Jersey (1988)

49. Jay, G.M., Willis, S.L.: Influence of direct computer experience on older adults' attitudes toward computers. Journal of Gerontology 47, 250--257 (1992)

50. Jian, J.Y., Bisantz, A.M., Drury, C.G.: Foundations for an empirically determined scale of trust in automated systems. International Journal of Cognitive Ergonomics 4, 53--71 (2000)

51. Mcknight, D.H., Carter, M., Thatcher, J.B., Clay, P.F.: Trust in a specific technology: An investigation of its components and measures. ACM Transactions on Management Information Systems (TMIS) 2, 12 (2011)

52. Gordon-Salant, S.: Hearing loss and aging: New research findings and clinical implications. Journal of Rehabilitation Research and Development 42, 9-24 (2005)

53. Browne, D., Totterdell, P., Norman, M.: Adaptive user interfaces. Elsevier, London, UK (1990)

54. Voinescu, A., Morgan, P.L., Caleb-Solly, P., Alford, C.: Investigating older adults' preferences for functions within a human-machine interface designed for fully autonomous vehicles. In: 20th International Conference on Human Computer Interaction 2018 (in press) 